\title{
ON THE THERMODYNAMICAL INTERPRETATION OF PERFECT FLUID SOLUTIONS OF THE EINSTEIN EQUATIONS WITH NO SYMMETRY
}

\author{
Andrzej Krasiński ${ }^{1}$, Hernando Quevedo ${ }^{2}$ and Roberto A. Sussman ${ }^{2}$ \\ ${ }^{1}$ N. Copernicus Astronomical Center, Polish Academy of Sciences \\ Bartycka 18, 00716 Warszawa, Poland \\ akr@alfa.camk.edu.pl \\ School of Sciences, Polish Academy of Sciences \\ Aleja Lotników 32/46, 02668 Warszawa, Poland \\ ${ }^{2}$ Instituto de Ciencias Nucleares \\ Universidad Nacional Autónoma de México
}

A. P. 70543, México, D. F. 04510, México

\begin{abstract}
The Gibbs - Duhem equation $d U+p d V=T d S$ imposes restrictions on the perfect fluid solutions of Einstein equations that have a one-dimensional symmetry group or no symmetry at all. In this paper, we investigate the restrictions imposed on the Stephani Universe and on the two classes of models found by Szafron. Upon the Stephani Universe and the $\beta^{\prime} \neq 0$ class of Szafron symmetries are forced. We find the most general subcases of the $\beta^{\prime}=0$ model of Szafron that are consistent with the Gibbs - Duhem equation and have no symmetry.
\end{abstract}

PACS Numbers: 04.20.-q; 98.80.k 


\section{The problem.}

In relativity, a perfect fluid is defined as a continuous medium whose state is determined by the energy-density $(\epsilon)$, the pressure $(p)$ and the four-velocity $\left(u^{\alpha}\right)$ fields, and whose energy-momentum tensor has the form:

$$
T_{\alpha \beta}=(\epsilon+p) u_{\alpha} u_{\beta}-p g_{\alpha \beta}
$$

(we will use the signature (+, -, -, -), Greek indices running through the values $0,1,2$, 3 and Latin indices running through the values 1,2,3). Indeed, this form of the energymomentum tensor guarantees that energy transport occurs only by means of mass-flow what is a characteristic property of perfect fluids. However, a single-component perfect fluid must also obey the appropriate laws of thermodynamics. One of them is the Gibbs Duhem equation which forms a part of the second law of thermodynamics. It says that the internal energy $U$, the pressure $p$, volume $V$, temperature $T$ and entropy $S$ of a thermally isolated portion of perfect fluid are connected by:

$$
d U+p d V=T d S .
$$

In relativity, and in particular in cosmology, we require that eq. (1.2) applies when the internal energy, volume and entropy are referred to one particle of the fluid. It is postulated that the particle number is a conserved quantity so that the particle number density $n$ obeys:

$$
\left(n u^{\alpha}\right)_{; \alpha}=0
$$

and then $U=\epsilon / n, V=1 / n$ and $S=s / n$, where $s$ is the entropy- density. The relativistic version of the equation (1.2) for a perfect fluid with the energy-momentum tensor (1.1) is thus:

$$
d(\epsilon / n)+p d(1 / n)=T d S .
$$

It is usually taken for granted that this equation applies. However, most of the perfect fluid solutions of the Einstein equations considered in the literature are thermodynamically trivial in one way or another. For dust, $p=0, \epsilon / n=$ const and $S=$ const, so (1.4) is no limitation. For perfect fluids with a barotropic equation of state $\epsilon=\epsilon(p)$, eq.(1.4) with $d S=0$ is a part of the definition of the solution. For cosmological models with a Robertson - Walker, Kantowski - Sachs or Bianchi-type geometries, all the functions $\epsilon, p$ and $n$ depend 
only on the comoving time $t$ of the fluid, so any equation of state can be imposed on them, and then (1.4) simply defines entropy. For solutions that have a symmetry group with twodimensional orbits (for example, the spherically symmetric ones), the functions $\epsilon, p$ and $n$ depend on two variables only. In this case, the left-hand side of (1.4) is a differential form in two variables and is guaranteed to have an integrating factor, hence $T$ and $S$ obeying (1.4) are guaranteed to exist.

Problems appear when the perfect fluid solution in question has a one-dimensional symmetry group or no symmetry at all. For such solutions, $\epsilon, p$ and $n$ depend on three or four variables, and the existence of an integrating factor for the left-hand side of (1.4) is an additional limitation on the state functions. This problem received only a fleeting attention in the literature (see below). In this paper, we shall consider the consequences of (1.4) for a few perfect fluid cosmological models with no symmetry. If $[d(\epsilon / n)+p d(1 / n)]$ has no integrating factor, then the solution can be interpreted as a mixture of perfect fluids (possibly interacting through reversible chemical reactions), but not as a single-component perfect fluid. If (1.3) and (1.4) can be imposed simultaneously, then, for brevity, we shall say that the model allows for a thermodynamical scheme.

Bona and Coll [1] were apparently the first to observe that (1.4) may lead to additional limitations for some solutions: they showed that the Stephani Universe [2 - 5] allows for a thermodynamical scheme only if the metric is restricted so that it acquires a 3-dimensional symmetry group acting on two- dimensional orbits. Relativistic thermodynamics of perfect fluids was discussed at length by Coll and Ferrando [6] without invoking explicit examples. Finally, Quevedo and Sussman [7] gave an example of the Szafron $\beta^{\prime}=0$ model [8] that has no symmetry and allows for a thermodynamical scheme, and showed that the parabolic Szafron $\beta^{\prime} \neq 0$ model does not allow for it unless it has a symmetry. Quevedo and Sussman [9] also analyzed the conditions for the existence of a thermodynamical scheme in the Stephani Universe, and derived the corresponding non barotropic equation of state. In this case the equation of state does not allow any plausible physical interpretation. This seems to be the whole body of literature on the subject published so far.

In this paper, we investigate the Szafron models systematically. We identify the most general Szafron models of the $\beta^{\prime}=0$ family that allow for a thermodynamical scheme (sec. 4) even though they have no symmetry, and show that the general Szafron models of the $\beta^{\prime} \neq 0$ family do not allow a thermodynamical scheme unless they have symmetries (sec. $5)$. These are the main results of the paper. In addition, in sec. 2 we discuss the necessary 
conditions for the existence of the thermodynamical scheme, and in sec. 3 we rederive the result of Bona and Coll [1] for the Stephani Universe by a different method and with some additional details.

The models of Stephani [2] and of Szafron [8] are so far the only known exact solutions of the Einstein equations that have no symmetry, can be considered to be cosmological models (because they generalize the Robertson - Walker class of solutions) and allow for nontrivial thermodynamics; see also Ref. [10]. The other class of solutions found by Stephani [11] has no symmetry as well, and has some cosmological relevance [10], but it has constant pressure. Therefore its source is in fact dust in a spacetime with cosmological constant, and (1.4) is trivially satisfied for it. The well-known Szekeres solutions [12] with no symmetry are the dust limit of the Szafron models considered here, and so pose no thermodynamical problems either.

\section{The necessary conditions for the existence of the thermodynamical scheme.}

Let us write (1.4) in the form:

$$
\omega:=(1 / n) d \epsilon-\left(1 / n^{2}\right)(\epsilon+p) d n=T d S .
$$

In the models we shall consider, the quantities $\epsilon, p$ and $n$ will be given functions of the coordinates, and so $\omega$ will be a given differential form in four variables (although the form is spanned on just two differentials, $d \epsilon$ and $d n$, the function $p$ will in general be functionally independent of $\epsilon$ and $n$ ). Eq. (2.1) can be solved for $T$ and $S$ if $\omega$ has an integrating factor, i.e. if $\omega \wedge d \omega=0$. This may be written equivalently as:

$$
d \epsilon \wedge d p \wedge d n=0
$$

which means that a functional dependence (an equation of state) connects $\epsilon, p$ and $n$.

Note that eq. (1.3) is a necessary, but not a sufficient condition for $n$ to be interpreted as a particle number density. The physical particle number density must obey a thermodynamically meaningful equation of state. In this paper, we shall not impose any condition on $n$ apart from (1.3). Therefore, only our negative results will be conclusive: if (1.3) and (2.2) imply additional symmetry, then the model does not allow for a thermodynamical scheme in general. If (1.3) and (2.2) can be imposed without introducing a 
symmetry, then additional work on the interpretation of $n$ is required. This we postpone to a separate paper.

The result of this paper is that for the Stephani models and the Szafron models with $\beta^{\prime} \neq 0$ a nontrivial thermodynamical scheme imposes symmetries, while the Szafron models with $\beta^{\prime}=0$ are restricted by the thermodynamical scheme in a different way which not necessarily implies a symmetry in general. (We call the thermodynamical scheme trivial if it implies $p=$ const, and in particular $p=0$ ).

\section{The thermodynamical scheme in the Stephani Universe.}

The metric of the Stephani Universe is:

$$
d s^{2}=D^{2} d t^{2}-V^{-2}(t, x, y, z)\left(d x^{2}+d y^{2}+d z^{2}\right)
$$

where:

$$
\begin{gathered}
V=R^{-1}\left\{1+\frac{1}{4} k(t)\left[\left(x-x_{0}(t)\right)^{2}+\left(y-y_{0}(t)\right)^{2}+\left(z-z_{0}(t)\right)^{2}\right]\right\} \\
D=F(t) V_{t} / V
\end{gathered}
$$

and $F(t), R(t), k(t), x_{o}(t), y_{o}(t)$ and $z_{o}(t)$ are arbitrary functions of time. The source in the Einstein equations is a perfect fluid with the velocity field $u^{\alpha}=D^{-1} \delta_{0}^{\alpha}$ and energydenstity $\epsilon$ and pressure $p$ given by:

$$
\begin{gathered}
\kappa \epsilon=3 C^{2}(t), \\
\kappa p=-3 C^{2}+2 C C,{ }_{t} V / V,{ }_{t},
\end{gathered}
$$

where $\kappa=8 \pi G / c^{4}$ and $C(t)$ is connected with the other functions of time by:

$$
k(t)=\left[C^{2}(t)-1 / F^{2}(t)\right] R^{2}(t) .
$$

The function $F(t)$ can be arbitrarily changed by transformations of the form $t=f\left(t^{\prime}\right)$, and $C(t)$ is determined by (3.4). Therefore, there are 5 arbitrary functions of time in (3.1). 
The Stephani Universe has in general no symmetry and is the most general conformally flat perfect fluid solution with nonzero expansion (see theorem 32.15 in Ref. [13]). A particle number density function obeying (1.3) has here the form:

$$
n=N(x, y, z) V^{3},
$$

where $N$ is an arbitrary function.

For reference, the following properties of the Stephani Universe should be noted:

I. When $\epsilon_{t}=0$, the Stephani Universe reduces to the de Sitter solution [3].

II. When $k, x_{0}, y_{0}$ and $z_{0}$ are all constant, the Stephani Universe reduces to the general Robertson - Walker (R-W) metric [3].

III. When $(V, t / V)$ is independent of any single variable of the set $\{x, y, z\}$, it is independent of all the variables $\{x, y, z\}$, and the Stephani Universe reduces to the R-W metric again (this is verified by a simple calculation).

IV. Property III applies to $\left(N V^{3}\right)$.

V. When $x_{0}, y_{0}, z_{0}$ are all constant, the Stephani Universe becomes spherically symmetric.

We shall refer to these as Properties I, .., V, respectively.

We shall now follow the consequences of (2.2) for the Stephani Universe and show that it necessarily implies symmetries. The equation (2.1) takes here the form:

$$
\omega \equiv\left(2 C C,{ }_{t} V / V,{ }_{t}\right)\left[1 /\left(N V^{3}\right)\right],{ }_{i} d x^{i}=T d S,
$$

where $x^{1}=x, x^{2}=y, x^{3}=z$. Since $\omega_{0}=0$, it follows that $S,_{t}=0$, i.e. that the entropy per particle is conserved along the fluid flow lines, as it must for a perfect fluid. Because of Properties I and IV we only need to investigate the case when all $\omega_{i} \neq 0$. Then the equation $\omega \wedge d \omega=0$ implies $\left(\omega_{i} / \omega_{j}\right)_{t}=0$, for all $i$ and $j$, i.e.:

$$
\begin{aligned}
& \left(N V^{3}\right),_{x} /\left(N V^{3}\right),_{y}=G_{3}(x, y, z), \\
& \left(N V^{3}\right){ }_{x} /\left(N V^{3}\right){ }_{z}=G_{2}(x, y, z),
\end{aligned}
$$

where $G_{3}$ and $G_{2}$ are arbitrary functions. Eq. (3.7) can be written in the equivalent form:

$$
N,_{x} / N+3 V,_{x} / V=G_{3}\left(N,_{y} / N+3 V,_{y} / V\right) .
$$

When differentiated by $t$ this becomes:

$$
(\ln V)_{, t x}=G_{3}(\ln V){ }_{t y} .
$$


Because of Property III we only need to investigate the case $(\ln V)_{, t x} \neq 0 \neq(\ln V)_{\text {, }}$, and then (3.10) implies:

$$
\left[(\ln V)_{, x} /(\ln V)_{, t y}\right]_{t}=0 .
$$

This is a necessary condition for the existence of a solution of (3.7), and it is an additional condition imposed on $V$. After being multiplied by $R^{3} V^{3}\left[(\ln V)_{, t y}\right]^{2}$, the left-hand side of (3.11) becomes a polynomial in $(x, y, z)$, and the coefficients of different powers of $x, y$ and $z$ have to vanish separately. This leads to differential equations imposed on the functions $k, x_{0}, y_{0}$ and $z_{0}$. From Property $V$ it follows that we only need to consider the case when at least one of the functions $x_{0}, y_{0}, z_{0}$ is not constant. Since the three functions can be permuted by relabelling $x, y$ and $z$, let us assume that $x_{0, t} \neq 0$. The equations resulting from (3.11) imply then:

$$
y_{0}=C_{1} x_{0}, \quad z_{0}=C_{2} x_{0},
$$

where $C_{1}$ and $C_{2}$ are arbitrary constants. Now it can be shown by a simple calculation that an orthogonal transformation among $\{x, y, z\}$ leads to $y_{0}=z_{0}=0$, so that:

$$
V=R^{-1}\left\{1+\frac{1}{4} k(t)\left[\left(x-x_{0}(t)\right)^{2}+y^{2}+z^{2}\right]\right\},
$$

With such $V$, eq. (3.11) reduces to:

$$
\frac{1}{2} k k,{ }_{t t} x_{0, t}-k,_{t}^{2} x_{0, t}-\frac{1}{2} k k,{ }_{t} x_{0, t t}-\frac{1}{4} k^{3} x_{0, t}^{3}=0 .
$$

One of the solutions of this is $x_{0}=$ const, but then the Stephani Universe becomes spherically symmetric. Another solution is $k=0$ in which case the Universe reduces to the flat $\mathrm{R}-\mathrm{W}$ model. Hence, we only need to investigate the case $k \neq 0 \neq x_{0, t}$, and then the solution of (3.13) is:

$$
1 / k=-\frac{1}{4} x_{0}^{2}+A x_{0}+B,
$$

where $A$ and $B$ are arbitrary constants. In this case, $V$ may be written in the form:

$$
V=\frac{k}{4 R}\left(x^{2}+y^{2}+z^{2}-2 x_{0} x+4 A x_{0}+4 B\right) .
$$

Only the rotational symmetry in the $(y, z)$-plane is evident here, but in fact this subcase of the Stephani Universe has a 3-dimensional symmetry group acting on 2-dimensional orbits 
(this result was found by Bona and Coll [1]). The generators of the group, found from the Killing equations, are:

$$
\begin{gathered}
k_{1}=\left(-\frac{1}{2} x y+A y\right) \frac{\partial}{\partial x}+\left[\frac{1}{4}\left(x^{2}-y^{2}+z^{2}\right)-A x-B\right] \frac{\partial}{\partial y}-\frac{1}{2} y z \frac{\partial}{\partial z}, \\
k_{2}=\left(-\frac{1}{2} x z+A z\right) \frac{\partial}{\partial x}-\frac{1}{2} y z \frac{\partial}{\partial y}+\left[\frac{1}{4}\left(x^{2}+y^{2}-z^{2}\right)-A x-B\right] \frac{\partial}{\partial z}, \\
k_{3}=z \frac{\partial}{\partial y}-y \frac{\partial}{\partial z},
\end{gathered}
$$

and the commutators among them are:

$$
\begin{gathered}
{\left[k_{1}, k_{2}\right]=\left(A^{2}+B\right) k_{3},} \\
{\left[k_{2}, k_{3}\right]=k_{1}, \quad\left[k_{3}, k_{1}\right]=k_{2} .}
\end{gathered}
$$

From here, it is seen that with $B>-A^{2}$ the solution is spherically symmetric, with $B<-A^{2}$ it is hyperbolically symmetric, and with $B=-A^{2}$ it is plane symmetric.

We shall show how the spherically symmetric case may be reduced to the standard form. Note that $A=0$ may be assumed with no loss of generality (this is achieved by the coordinate transformation $x=x^{\prime}+2 A$ toghether with the redefinitions $x_{0}=x_{0}^{\prime}+2 A, B=$ $\left.B^{\prime}-A^{2}\right)$. Then the solution is spherically symmetric when $B>0$, and the following sequence of coordinate transformations reduces it to the standard spherically symmetric form:

$$
x=\left[x^{\prime}+(4 B)^{-1 / 2}\left(x^{\prime 2}+y^{\prime 2}+z^{\prime 2}\right)\right] / W, \quad y=y^{\prime} / W, \quad z=z^{\prime} / W,
$$

where

$$
W:=1+2(4 B)^{-1 / 2} x^{\prime}+(4 B)^{-1}\left(x^{\prime 2}+y^{\prime 2}+z^{\prime 2}\right)
$$

(the above is a conformal symmetry of the Euclidean 3-space),

$$
x^{\prime}=x^{\prime \prime}-B^{1 / 2} .
$$

It may be useful to note that with $V$ given by (3.15), eqs. (3.7) and (3.8) can be solved. The most general form of the function $N$ that obeys them is:

$$
N=C G(w) /(x-2 A)^{3},
$$


where $C$ is an arbitrary constant, $G$ is an arbitrary function, and:

$$
w:=x+\left(y^{2}+z^{2}+4 A^{2}+4 B\right) /(x-2 A) .
$$

Note that $N$ given by (3.20) in general has a time-independent singularity or a zero at $x=2 A$ unless $G=$ const $/ w^{3}$, which is another warning that not every $n$ obeying (1.3) has a physical interpretation. Note also that $w$ and $n=N V^{3}$ given by (3.21), (3.20) and (3.15) are both invariant under all the three generators (3.16), i.e. $k_{i}^{\alpha} w,_{\alpha}=k_{i}^{\alpha} n,_{\alpha}=0, i=1,2,3$.

The result (3.20) - (3.21) is equivalent to the one by Bona and Coll [1] except that Bona and Coll obtained it by postulating invariance of $n$ with respect to (3.16), while we showed that (3.20) - (3.21) is a general solution of the conditions for a thermodynamical scheme, and invariance necessarily follows. In Ref. [9], the thermodynamical scheme conditions for a special case of the solution (3.15) were investigated. Using a work by Bona and Coll [14], Quevedo and Sussman claim that their special solution admits only a one-parameter group of isometries. This result is false as, according to the general result presented here in (3.16), that special solution must admit a 3-dimensional group of isometries.

\section{The thermodynamical scheme in the Szafron models with $\beta^{\prime}=0$.}

The metric of the Szafron models with $\beta^{\prime}=0$ is:

$$
d s^{2}=d t^{2}-e^{2 \alpha} d z^{2}-e^{2 \beta}\left(d x^{2}+d y^{2}\right)
$$

where:

$$
\begin{gathered}
e^{\beta}=\Phi(t) /\left[1+\frac{1}{4} k\left(x^{2}+y^{2}\right)\right], \\
e^{\alpha}=\lambda(t, z)+S e^{\beta}, \\
S=\left[\frac{1}{2} U(z)\left(x^{2}+y^{2}\right)+V_{1}(z) x+V_{2}(z) y+2 W(z)\right],
\end{gathered}
$$

$k$ is an arbitrary constant, $U(z), V_{1}(z), V_{2}(z)$ and $W(z)$ are arbitrary functions, the function $\Phi(t)$ is determined by the equation:

$$
2 \Phi, t t / \Phi+\Phi,{ }_{t}^{2} / \Phi^{2}+k / \Phi^{2}+\kappa p(t)=0
$$

where $p(t)$ (the pressure in the perfect fluid source) is an arbitrary function, and the function $\lambda(t, z)$ is determined by the equation:

$$
\Phi \lambda_{t t}+\Phi,_{t} \lambda,_{t}+\left(\Phi,{ }_{t t}+\kappa p \Phi\right) \lambda=U(z)+k W(z)
$$


Eq. (4.6) can be written in the equivalent form:

$$
\Phi \lambda,_{t t}+\Phi,{ }_{t} \lambda, t-\left(\Phi, t t+\Phi,{ }_{t}^{2} / \Phi+k / \Phi\right) \lambda=U(z)+k W(z)
$$

The source in the Einstein equations is a geodesically and irrotationally moving perfect fluid with the pressure $p(t)$, the velocity field $u^{\alpha}=\delta_{0}^{\alpha}$, and the energy-density given by:

$$
\kappa \epsilon=2 E(t, z) e^{-\alpha}+3\left(\Phi,{ }_{t}^{2}+k\right) / \Phi^{2},
$$

where:

$$
E(t, z):=\lambda \Phi,{ }_{t t} / \Phi-\lambda, t t \equiv \Phi,_{t} \lambda, t / \Phi-\left(\Phi,{ }_{t}^{2}+k\right) \lambda / \Phi^{2}-(U+k W) / \Phi
$$

The $\beta^{\prime}=0$ means that $\beta$ does not depend on $z$; this case has to be considered separately because the limit $\beta, z \rightarrow 0$ of the corresponding solutions with $\beta, z \neq 0$ is singular, see sec. 5. An overview of properties of these solutions, along with a complete list of literature about them, is given in Ref. [10]. The $\beta^{\prime}=0$ solutions simultaneously generalize the Robertson - Walker (R-W) metrics (which result when $\lambda=0$ and $U=-k W, k$ is the spatial curvature index in the limit) and metrics with the Kantowski - Sachs (K-S) symmetry [10, 15 - 16] (which result when $U=V_{1}=V_{2}=W=\lambda, z=0$ ). For reference, the following properties of these metrics should be noted:

I. When $p=$ const, the solution reduces to dust with cosmological constant, whose thermodynamics is trivial. Note that the subcase $\Phi_{, t}=0$ necessarily leads to $p=$ const.

II. When $E=0$, the solution becomes spatially homogeneous (with either R-W or K-S or Bianchi-type symmetry) [17].

III. When $(\lambda / \Phi)_{t}=0$, shear is zero and the solution reduces to an $\mathrm{R}-\mathrm{W}$ metric.

We shall refer to these as Properties I, II and III, respectively.

With no loss of generality, we can assume $W(z)=0$. This specialization amounts to just redefining $U$ and $\lambda$ (see Ref. 10), and we shall do so in the following. (After such a specialization the R-W limit changes to $\{U=-k u(z), \lambda=\Phi u\})$.

Note that the coordinate $z$ is not defined uniquely. All the formulae given are covariant with the transformations $z=f\left(z^{\prime}\right)$, where $f$ is an arbitrary function. We shall make use of this in Appendix B.

The particle number density function obeying (1.3) has here the form:

$$
n=N(x, y, z) e^{-\alpha-2 \beta},
$$


where $N(x, y, z)$ is an arbitrary function. Eq. (2.2) implies $p_{,}\left[(1 / n),{ }_{i} \epsilon,,_{j}-(1 / n),{ }_{j} \epsilon,_{i}\right]=$ $0, i, j=1,2,3$. Because of Property I we only need to consider the case $p_{t} \neq 0$, and so a nontrivial thermodynamical scheme will exist when:

$$
(1 / n),_{i} \epsilon,_{j}-(1 / n),{ }_{j} \epsilon,_{i}=0
$$

Substituting for $\epsilon, n$ and $e^{\alpha}$ from (4.10), (4.8) - (4.9) and (4.3) we obtain:

$$
\begin{gathered}
E,_{[i}(1 / N),{ }_{j]} e^{2 \beta}\left(\lambda+S e^{\beta}\right)-\left(\lambda+S e^{\beta}\right),{ }_{[i}(1 / N),{ }_{j]} E e^{2 \beta} \\
+E,_{[i}\left(\lambda+S e^{\beta}\right){ }_{j]}(1 / N) e^{2 \beta}+2 E,{ }_{[i}\left(e^{\beta}\right),{ }_{j]}(1 / N) e^{\beta}\left(\lambda+S e^{\beta}\right) \\
-2\left(\lambda+S e^{\beta}\right),{ }_{[i}\left(e^{\beta}\right),{ }_{j]} E(1 / N) e^{\beta}=0,
\end{gathered}
$$

square brackets around indices denoting antisymmetrization.

Now let $x^{1}=z, x^{2}=x, x^{3}=y$, and let us take $i=2, j=3$ in (4.12). Since $E$ and $\lambda$ are independent of $x$ and $y$, the equation simplifies to:

$$
\begin{aligned}
& -E e^{\beta}\left\{e^{\beta}\left[(1 / N),_{y}\left(S e^{\beta}\right),_{x}-(1 / N),{ }_{x}\left(S e^{\beta}\right), y\right]\right. \\
& \left.+(2 / N)\left[\left(e^{\beta}\right),_{y}\left(S e^{\beta}\right),_{x}-\left(e^{\beta}\right),{ }_{x}\left(S e^{\beta}\right),{ }_{y}\right]\right\}=0 .
\end{aligned}
$$

Because of Property II we do not need to consider the case $E=0$. Noting that:

$$
e^{B}:=e^{\beta} / \Phi
$$

is independent of time we see that $\Phi$ factors out in (4.13), and what remains is a quasilinear partial differential equation determining $(1 / N)$. There are no derivatives by $z$ in (4.13), so the dependence of $N$ on $z$ is not determined by (4.13). The general solution of (4.13) is thus:

$$
N=e^{2 B} F\left(S e^{B}, z\right),
$$

where $F$ is an arbitrary function of its two arguments.

Now let us take $i=1, j=2$ in (4.12) and let us substitute (4.15) into it. The result is:

$$
\left(S e^{B}\right),_{x}\left\{-E,_{z}\left[\left(\lambda+S e^{\beta}\right) F,_{I} / F-\Phi\right]+E\left(\lambda,{ }_{z} F,_{I} / F-\Phi F,_{I I} / F\right)\right\}=0 .
$$

where $F,_{I}$ and $F,_{I I}$ are partial derivatives of $F$ with respect to its first and second argument, respectively. Taking $i=1, j=3$ in (4.12) we would obtain an equation of the same form 
as (4.16), with $\left(S e^{B}\right){ }_{y}$ in place of $\left(S e^{B}\right),_{x}$. One way of fulfilling these two equations is $\left(S e^{B}\right){ }_{x}=\left(S e^{B}\right),{ }_{y}=0$. However, this implies $U=V_{1}=V_{2}=0$, and the resulting solution has a 3-dimensional symmetry acting on 2-dimensional orbits; the thermodynamics of this case poses no problems. Hence, we may assume that either $\left(S e^{B}\right),_{x}$ or $\left(S e^{B}\right),_{y}$ is nonzero, and then we can define the following new variables in (4.16):

$$
X=S e^{B}, \quad Z=z
$$

We have then from (4.16):

$$
\left[(\lambda / \Phi+X) E,_{Z}-E \lambda,_{Z} / \Phi\right] F,_{X} / F+E F,_{Z} / F=E,_{Z}
$$

This is again a quasi-linear partial differential equation determining $F(X, Z)$. However, the coefficients in (4.18) do depend on time, while $F$ should be, by the definition of $N$, independent of $t$. We shall first solve (4.18) as if $F$ were allowed to depend on $t$, and then we will impose the condition $F_{t}=0$. The general solution of (4.18) is:

$$
F=E G(\mathcal{U})
$$

where $G$ is an arbitrary function and:

$$
\mathcal{U}:=(\lambda / \Phi+X) / E+f(t)
$$

$f(t)$ being another arbitrary function. The condition $F_{,}=0$ reads now:

$$
E,_{t} G+G, \mathcal{U}\left[(\lambda / \Phi){ }_{t}-(\lambda / \Phi+X) E,_{t} / E+f{ }_{t} E\right]=0
$$

Three cases arise here:

$$
\text { I. } E_{t}=0=G, \mathcal{U} \text {. }
$$

This will turn out to be included in the case III below and does not require a separate treatment.

$$
\text { II. } E, t=0, \quad(\lambda / \Phi), t=-f,{ }_{t} E \text {. }
$$

This one will be considered separately further on.

$$
\text { III. } E, t \neq 0 \neq G, \mathcal{U} \text {. }
$$


This is the generic case that we will consider first. Then (4.21) can be rewritten in the form:

$$
G / G, \mathcal{U}-\mathcal{U}=-(\lambda / \Phi){ }_{,} / E,_{t}-f-f{ }_{t} E / E,_{t} .
$$

Since the right-hand side of this is independent of $X$, this implies $G, \mathcal{U U}=0$, i.e.:

$$
F=A(\lambda / \Phi+X)+\psi(t) E,
$$

where $A$ is an arbitrary constant and $\psi(t)$ is an arbitrary function. Now it is seen that the case $E_{,}=0=G_{, \mathcal{U}}$ is contained here as the subcase $A=0=E_{, t}=\psi_{, t}$. We can assume $\psi \neq 0$ because with $\psi=0$ the condition $F_{, t}=0$ implies either $A=F=0$, i.e. $n=0-a$ thermodynamical nonsense, or $(\lambda / \Phi)_{t}=0$, i.e. an R-W metric (see Property III). The condition $F, t=0$ implies now:

$$
A \lambda / \Phi+\psi E=H(z)
$$

where $H(z)$ is an arbitrary function. Substituting the definition of $E$ into this we obtain:

$$
\lambda_{, t}=K(t) \lambda+L(t, z),
$$

where:

$$
\begin{gathered}
K(t):=\Phi_{, t} / \Phi+k /(\Phi \Phi, t)-A /\left(\psi \Phi,_{,}\right), \\
L(t, z):=U(z) / \Phi,_{t}+H(z) \Phi /\left(\psi \Phi,_{t}\right) .
\end{gathered}
$$

(Because of Property I we may assume $\Phi_{, t} \neq 0$ ). The solution of eq. (4.25) is:

$$
\lambda=J(z) e^{\int K d t}+e^{\int K d t} \int L e^{-\int K d t} d t,
$$

where $J(z)$ is another arbitrary function of $z$. The $\lambda$ given by (4.28) must now be compatible with the definition of $\lambda$, eq. (4.7) (recall: we assumed $W=0$ ). When (4.28) is substituted into (4.7), an equation of the following form results:

$$
J(z) F_{1}(t)+U(z) F_{2}(t)+H(z) F_{3}(t)=0,
$$

where $F_{1}, F_{2}$ and $F_{3}$ are functions of $t$ composed of $\Phi, \psi$ and their derivatives (see Appendix A). Three possibilities now arise: 
( $\alpha$ ) All the three functions $J, U$ and $H$ are linearly independent. Then (4.29) implies $F_{1}=F_{2}=F_{3}=0$. With $F_{1}=0$ assumed, $F_{2}=0$ implies:

$$
-\Phi \Phi,{ }_{t t}+\Phi,{ }_{t}^{2}+k-A \Phi / \psi=0 .
$$

With $F_{1}=F_{2}=0$ taken into account, $F_{3}=0$ implies:

$$
2 \Phi / \psi-\Phi^{2} \psi,{ }_{t} /\left(\psi^{2} \Phi,{ }_{t}\right)=0 .
$$

This integrates to $\psi=b \Phi^{2}, b=$ const, and with this (4.30) has the first integral:

$$
\Phi,{ }_{t}^{2}=-k+\frac{2}{3} A /(b \Phi)+C \Phi^{2},
$$

where $C$ is an arbitrary constant. With (4.31) fulfilled, $F_{1}=0$ follows. However, (4.31) together with (4.6) implies $\kappa p=-3 C=$ const, so this case is thermodynamically trivial.

( $\beta$ ) Two of the functions $\{J, U, H\}$ are linearly independent, while the third one is their linear combination. In each of these cases, two linear combinations of the functions $\left\{F_{1}, F_{2}, F_{3}\right\}$ must vanish, which leads to a set of two differential equations to be obeyed by $\psi(t)$ and $\Phi(t)$. Some of the resulting solutions are nontrivial, but not all of them. For example, in the two cases:

$$
\begin{gathered}
(\beta i) H=0,\{J, U\}-\text { linearly independent; } \\
(\beta i i) U=0,\{J, H\}-\text { linearly independent, } k \neq 0 ;
\end{gathered}
$$

the trivial solution from point $(\alpha)$ reappears. However, with $U=k=0$ and $\{J, H\}$ being linearly independent, the functions $\Phi$ and $\psi$ have to obey only one equation:

$$
\Phi,{ }_{t t}=-A / \psi+3 \Phi,{ }_{t}^{2} / \Phi-\psi,{ }_{t} \Phi,{ }_{t} / \psi,
$$

which means that $\Phi(t)$ can be arbitrary, $p(t)$ (in general nontrivial) is determined by (4.5) with $k=0$, and $\psi(t)$ is determined by (4.33). In Appendix $B$ we show under what conditions the Szafron model with $\beta^{\prime}=0$ has symmetries. It follows that with $U=k=0,(\lambda / \Phi)_{t} \neq 0$ and generic $V_{1}$ and $V_{2}$ the model has no symmetry. Hence, the case we just identified is an example of a Szafron $\beta^{\prime}=0$ model with no symmetry that allows for a thermodynamical scheme. 
$(\gamma)$ Each pair in the set $\{J, U, H\}$ is linearly dependent. This means that there is only one function of $z$ in this set, let us call it $f(z)$, and:

$$
J=j f, \quad U=u f, \quad H=h f,
$$

where $j, u$ and $h$ are arbitrary constants. In this case, $\Phi$ and $\psi$ are connected by just one equation,

$$
j F_{1}+u F_{2}+h F_{3}=0,
$$

i.e. $\Phi(t)$ is again arbitrary, $p(t)$ is given by (4.5) and $\psi(t)$ is given by (4.35). It follows from Appendix $B$ that the Szafron model has in general no symmetry also in this case, so we have here another example of a model with a thermodynamical scheme and with no symmetries.

The case discussed by Quevedo and Sussman [7] is contained in the class $(\gamma)$ as the subcase $j=h=F_{2}=0$. This is shown in Appendix C.

Note that in the cases $(\beta)$ and $(\gamma)$ above, the function $\lambda$ is more special than eq. (4.7) would allow. The general solution of (4.7) has the structure:

$$
\lambda=\mathcal{F}_{1}(t) \mathcal{G}_{1}(z)+\mathcal{F}_{2}(t) G_{2}(z)+\mathcal{F}_{3}(t, z)
$$

where $\mathcal{F}_{3}(t, z)$ is a specific solution of the full inhomogeneous $(4.7), \mathcal{F}_{1}(t)$ and $\mathcal{F}_{2}(t)$ are the basis solutions of the homogeneous part of $(4.7)$ and $\mathcal{G}_{1}(z)$ and $\mathcal{G}_{2}(z)$ are arbitrary functions. This means that a general Szafron model with $\beta^{\prime}=0$ contains two arbitrary functions of $z$ hidden in $\lambda$ in addition to the set $\left\{U, V_{1}, V_{2}\right\}$. By contrast, in the case $(\beta)$ above there is only one additional function of $z$ in $\lambda$, and in the case $(\gamma)$ with $u \neq 0$ there are none; $U$ determines both of them.

It remains now to investigate the case II of the three cases listed after eq. (4.21). We have then $E, t=0$ and:

$$
\lambda=-E(z) f(t) \Phi(t)+H(z) \Phi(t),
$$

where $E(z)$ and $H(z)$ are arbitrary functions. This $\lambda$ must obey (4.7) with $W=0$ and (4.9) with $E=E(z)$. In this case, $F$ given by (4.19) and (4.20) is automatically independent of $t$. This subcase has nonzero shear as long as $f_{, t} \neq 0$ (if $f,,_{t}=0$, then the thermodynamically trivial solution with $\Phi_{,}=0$ results, see Property I). Eqs. (4.37), (4.7) and (4.9) lead to the following two equations determining $\Phi$ and $f$ :

$$
f_{, t}=k f /\left(\Phi \Phi,{ }_{t}\right)-1 / \Phi,_{t}-C /\left(\Phi \Phi,_{t}\right)
$$




$$
\Phi(\Phi+C) \Phi,{ }_{t t}-k(\Phi+C)-(3 \Phi+C) \Phi_{,}^{2}=\left(-k+\Phi \Phi, t t-\Phi,_{t}^{2}\right) k f
$$

where $C$ is an arbitrary constant. The functions $E, H$ and $U$ are connected by:

$$
k H+U=C E .
$$

When $k=0$, the equation (4.39) decouples from (4.38). Even in that special case, $p$ is not constant and the solution has in general no symmetry. Hence, this is another example of a Szafron $\beta^{\prime}=0$ model without a symmetry and with a nontrivial thermodynamical scheme.

\section{The thermodynamical scheme in the Szafron models with $\beta^{\prime} \neq 0$.}

The metric of these models is of the same form as in the previous case:

$$
d s^{2}=d t^{2}-e^{2 \alpha} d z^{2}-e^{2 \beta}\left(d x^{2}+d y^{2}\right)
$$

but here (in a notation adapted from Szafron [8]):

$$
\begin{gathered}
e^{\beta}=\Phi(t, z) / S(x, y, z), \\
e^{\alpha}=h(z) S \cdot\left(e^{\beta}\right), z \\
S:=A(z)\left(x^{2}+y^{2}\right)+2 B_{1}(z) x+2 B_{2}(z) y+C(z),
\end{gathered}
$$

where $h, A, B_{1}, B_{2}$ and $C$ are arbitrary functions of $z$, the function $\Phi(t, z)$ is determined by the equation:

$$
2 \Phi, t t / \Phi+\Phi,_{t}^{2} / \Phi^{2}+k(z) / \Phi^{2}+\kappa p(t)=0
$$

the function $p(t)$ (the pressure in the perfect fluid source) is arbitrary, and the function $k(z)$ must obey:

$$
A C-B_{1}^{2}-B_{2}^{2}=\frac{1}{4}\left[h^{-2}(z)+k(z)\right] .
$$

Note that the limit $\beta,{ }_{z} \rightarrow 0$ of this solution is singular. Therefore the case $\beta,,_{z}=0$ discussed in sec. 4 has to be derived separately from the Einstein equations.

The source in the Einstein equations is again a geodesically and irrotationally moving perfect fluid with the velocity field $u^{\alpha}=\delta_{0}^{\alpha}$, the pressure $p(t)$ and the energy-density:

$$
\kappa \epsilon=\left(h / \Phi^{2}\right) E(t, z) e^{-\alpha}+3\left(\Phi,{ }_{t}^{2}+k\right) / \Phi^{2} \equiv
$$




$$
\equiv E(t, z) /\left[\Phi^{3}(\Phi, z / \Phi-S, z / S)\right]+3\left(\Phi,{ }_{t}^{2}+k\right) / \Phi^{2}
$$

where this time:

$$
E(t, z)=\Phi\left(\Phi,{ }_{t}^{2}+k\right),_{z}-2 \Phi, z\left(\Phi,{ }_{t}^{2}+k\right)
$$

(This $E$ is not to be confused with the one from sec. 4; it plays the same role in $\epsilon$, but has a very different form here).

The R-W limit results when:

$$
\Phi=z R(t), \quad k=k_{0} z^{2}
$$

where $k_{0}$ is a constant (the spatial curvature index of the R-W metric) $[10,19]$.

The particle number density function defined by (1.3) is here:

$$
n=N(x, y, z) e^{-\alpha-2 \beta} \equiv N S^{2} /\left[h \Phi^{3}\left(\Phi,_{z} / \Phi-S,_{z} / S\right)\right]
$$

For reference, the following properties should be noted:

I. When $E=0$, the model reduces either to an R-W metric or to a vacuum solution with a cosmological constant.

II. When all the functions $A, B_{1}, B_{2}$ and $C$ are constant multiples of the same function $f(z)$, the model acquires a 3-dimensional symmetry group acting on 2-dimensional surfaces.

III. When $(\ln \Phi)_{t z}=0$, shear becomes zero and the model becomes an R-W metric $[10]$.

Since $p=p(t)$, just as in sec. 4, the condition of integrability of the Gibbs - Duhem equation has here the form:

$$
(1 / n),{ }_{i} \epsilon,{ }_{j}-(1 / n),{ }_{j} \epsilon,,_{i}=0,
$$

with $i, j=1,2,3, x^{1}=z, x^{2}=x, x^{3}=y$. When $i=2, j=3$, this becomes:

$$
E\left[-S,_{z}\left(N,_{x} S,_{y}-N,_{y} S,_{x}\right)+S\left(N,_{x} S,_{y z}-N,_{y} S,_{x z}\right)+2 N\left(S,_{x} S,_{y z}-S,_{y} S,_{x z}\right)\right]=0
$$

Because of Property I we are only interested in the case $E \neq 0$. The solution of (5.12) is thus:

$$
N=\left(h / S^{2}\right) F\left(S,_{z} / S ; z\right),
$$

where $F$ is an arbitrary function of the two arguments indicated. 
Taking (5.11) with $i=1, j=2$ and using (5.10) and (5.13) in the result we obtain:

$$
\begin{aligned}
& \Phi^{-6}(\ln S),_{x z}\left(\Phi,_{z} / \Phi-S,_{z} / S\right)^{-3}\left\{-E F,_{I I}+E,_{z}\left[\left(\Phi,_{z} / \Phi-S,_{z} / S\right) F,_{I}+F\right]\right. \\
& +3 E\left[\left(\Phi,_{z} / \Phi-S,_{z} / S\right)^{2} F,_{I}+\left(\Phi,_{z} / \Phi-S,_{z} / S\right) F\right] \\
& \left.+E\left[-2 \Phi^{-1} \Phi,_{z}\left(\Phi,_{z} / \Phi-S,_{z} / S\right)-\Phi^{-1}\left(\Phi, z z-\Phi,_{z} S,_{z} / S\right)\right] F,_{I}\right\}=0,
\end{aligned}
$$

where $F,_{I}$ and $F, I I$ are the partial derivatives of $F$ with respect to its arguments.

If $(\ln S),_{x z}=0$, then all the functions $A, B_{1}, B_{2}$ and $C$ are constant multiples of the same function of $z$, i.e. Property II applies. Hence, we are only interested in the case $(\ln S),_{x z} \neq 0$.

With $i=1, j=3$, eq. (5.11) would lead to (5.14) with $(\ln S),_{x z}$ replaced by $(\ln S){ }_{y z}$. We do not need to consider the case $(\ln S)_{y z}=0$ for the same reason as above. Hence, the resulting equation has the same solutions as (5.14), and so (5.14) is the last equation that $F$ has to obey.

When $(\ln S)_{x z} \neq 0 \neq(\ln S)_{y z}$, we can introduce the new variables:

$$
X=S,_{z} / S, \quad Z=z
$$

because $\frac{\partial(X, Z)}{\partial(x, z)} \neq 0 \neq \frac{\partial(X, Z)}{\partial(y, z)}$. In the new variables, eq.(5.14) becomes:

$$
\begin{gathered}
-F,_{Z} / F+\left[\left(\Phi,_{Z} / \Phi-X\right) E,_{Z} / E-3\left(\Phi,_{Z} / \Phi\right) X+3 X^{2}-\left(\Phi,_{Z} / \Phi\right),_{Z}\right] F,_{X} / F \\
+E,_{Z} / E+3(\Phi, Z / \Phi-X)=0 .
\end{gathered}
$$

We differentiate this by $t$ and use the fact that $F, t=0$. In this way we obtain a necessary condition for the existence of solutions of (5.16):

$$
\begin{gathered}
{\left[\left(\Phi,,_{Z} / \Phi\right),_{t} E,_{Z} / E+\left(\Phi,_{Z} / \Phi-X\right)\left(E,_{Z} / E\right),_{t}-3\left(\Phi,_{Z} / \Phi\right),_{t} X-\left(\Phi,_{Z} / \Phi\right),,_{t}\right] F,_{X} / F} \\
+\left(E,_{Z} / E\right),_{t}+3(\Phi, z / \Phi),_{t}=0
\end{gathered}
$$

The case when the coefficient of $(F, X / F)$ in (5.17) is zero must be considered separately. It is shown in Appendix $D$ that it leads to the $\mathrm{R}-\mathrm{W}$ metrics. It is also shown there that $(E, Z / E){ }_{t}+3(\Phi, Z / \Phi),_{t}=0=F,_{X}$ leads to a contradiction. Knowing this, we multiply (5.17) by $(F / F, X)$ and differentiate the result by $X$. We obtain:

$$
-\left[\left(E,_{Z} / E\right){ }_{t}+3\left(\Phi,,_{Z} / \Phi\right){ }_{t}\right] F F,_{X X} / F,_{X}^{2}=0 .
$$


The only case that must be investigated is $F,_{X X}=0$, i.e.:

$$
F=g_{1}(Z) X+g_{2}(Z)
$$

With this, eq. (5.16) becomes an explicit expression in $X$ with coefficients depending on $t$ and $Z$. It implies:

$$
\begin{gathered}
g_{2}=-\frac{1}{3} g_{1, Z}, \\
-g_{2, Z}+[E, Z \Phi, Z /(E \Phi)-(\Phi, Z / \Phi), Z] g_{1}+(E, Z / E+3 \Phi, Z / \Phi) g_{2}=0 .
\end{gathered}
$$

The solution of this set is:

$$
g_{1}(Z)=-3 A(t) \Phi\left(\Phi_{t}^{2}+k\right)-B(t) \Phi^{3}
$$

where $A(t)$ and $B(t)$ are arbitrary functions. This is an additional equation imposed on $\Phi$ that must be consistent with (5.5). The two equations turn out to be consistent only if either the source is dust or the model reduces to an R-W metric - this is shown in Appendix E. Hence, for this class of Szafron models a nontrivial thermodynamical scheme can exist only if there are symmetries in the spacetime.

\section{Conclusions.}

In this paper, we have proven the following:

1. For the Stephani Universe and the Szafron models with $\beta^{\prime} \neq 0$ a nontrivial thermodynamical scheme (that is, one in which $p \neq 0$ ) can exist only in those subcases in which the spacetime acquires an at least 3-dimensional symmetry group acting on at least 2-dimensional orbits.

2. The Szafron model with $\beta^{\prime}=0$ does have subcases that have no symmetry and allow for a nontrivial thermodynamical scheme. In the subcase of class $(\gamma)$ in sec. 4, the scale factor $\Phi(t)$ remains arbitrary, but the form of the function $\lambda$ is limited (the functions $\mathcal{G}_{1}$ and $\mathcal{G}_{2}$ from (4.36) and $U(z)$ are all constant multiples of the same function). In the nontrivial subcases of class $(\beta)$, the evolution of the scale factor is fixed, while the generality of $\lambda$ is limited to a lesser degree (there are two linearly independent functions in the set $\left.\left\{\mathcal{G}_{1}, \mathcal{G}_{2}, U\right\}\right)$. 
Like we stated in the introduction, only the first result is conclusive. For the Szafron model with $\beta^{\prime}=0$ it remains to be verified whether among the functions $n$ given by (4.10), (4.15), (4.23), (4.24) and (4.26) - (4.29) there are any which obey an interpretable equation of state.

Also, as stated in the introduction, the negative result of point 1 above only means that these models cannot be interpreted as single-component perfect fluids. It remains to be seen whether they can be interpreted as noninteracting mixtures of perfect fluids or mixtures in which reversible chemical reactions occur.

Our results show that there is no simple connection between the existence of a thermodynamical scheme and symmetries.

The hope that motivated this paper was that the Gibbs - Duhem equation (1.2) would force a definite form upon the arbitrary functions of time in the models, and thus would play a similar role as the equation of state does. This happens indeed in the class $(\beta)$ models of sec. 4 , but it is not a general rule. In the class $(\gamma)$ models of section 4 , the one arbitrary function of time, $p(t)$, survives intact after the integrability of (1.2) is ensured. In those cases where the Gibbs - Duhem equation imposes symmetries, arbitrary functions survive, too, but this was to be expected, since a high enough symmetry guarantees the existence of a nontrivial thermodynamical scheme irrespectively of the evolution law (see sec. 1). Hence, in the Stephani Universe, the Gibbs - Duhem equation reduces the number of arbitrary functions of $t$ from five to at most two, just the number compatible with a 3-dimensional symmetry acting on 2-dimensional orbits.

In those classes in which arbitrary functions of time are present in spite of the lack of symmetry, an equation of state still has to be imposed on the resulting solution. We recall (see Ref. 17) that for the Szafron model the barotropic equation of state $p=p(\epsilon)$ trivializes it to a spatially homogeneous one (and in particular to an $R-W$ one in some cases).

\section{Acknowledgments}

A.K. wishes to thank H.Q. and R.S. for their kind hospitality at the Instituto de Ciencias Nucleares of the UNAM where this collaboration was initiated. This work, including A.K.'s visit to Mexico, was supported by CONACYT grant No. 3567-E.

The calculations for this paper were partly done with the algebraic computer systems Maple [20] and Ortocartan [21]. 


\section{Appendix A}

The three functions appearing in eqs. (4.29) - (4.35) are defined as follows:

$$
\begin{aligned}
& F_{1}(t)=\left[k / \Phi-k \Phi,{ }_{t t} / \Phi{ }_{t}^{2}+A \Phi \psi,{ }_{t} /\left(\psi^{2} \Phi,{ }_{t}\right)+A \Phi \Phi,{ }_{t t} /\left(\psi \Phi,{ }_{t}^{2}\right)\right. \\
& \left.+(k / \Phi-A / \psi)^{2} \Phi / \Phi,{ }_{t}^{2}-3 A / \psi\right] e^{\int K d t} \\
& F_{2}(t)=F_{1}(t) \int\left(1 / \Phi,{ }_{t}\right) e^{-\int K d t} d t+1+k / \Phi,{ }_{t}^{2}-A \Phi /\left(\psi \Phi{ }_{t}^{2}\right)-\Phi \Phi,{ }_{t t} / \Phi,{ }_{t}^{2}, \\
& F_{3}(t)=F_{1}(t) \int[\Phi /(\psi \Phi, t)] e^{-\int K d t} d t+(\Phi / \psi)\left[1+k / \Phi,_{t}^{2}\right. \\
& \left.-A \Phi /\left(\psi \Phi,{ }_{t}^{2}\right)-\Phi \Phi,{ }_{t t} / \Phi,{ }_{t}^{2}\right]+2 \Phi / \psi-\Phi^{2} \psi,{ }_{t} /\left(\psi^{2} \Phi,{ }_{t}\right),
\end{aligned}
$$

where $K(t)$ is given by (4.26).

\section{Appendix B}

Conditions for the existence of symmetries in the Szafron models with $\beta^{\prime}=0$.

It was already shown by Bonnor, Sulaiman and Tomimura [18] that the Szekeres solutions in general have no symmetry. Since all the Szekeres solutions are contained in the Szafron models as the subcase $p=0$, this result immediately carries over to the latter. However, for the considerations of this paper it is important to know, exactly which subcases have symmetries. Bonnor et al. [18] did not make this completely clear, and their derivation relies on the property $p=0$. Therefore we present another derivation here.

For a perfect fluid solution, the pressure must be invariant under all the existing symmetries, i.e.:

$$
k^{\mu} p_{\mu}=0
$$

where $k^{\mu}$ is the general Killing vector field. Since in the Szafron models, in the comoving coordinates used here, $p$ depends only on $t$, and the case $p=$ const is thermodynamically trivial, we are interested only in the case $p_{,} \neq 0$. Hence, from (B.1):

$$
k^{0}=0 \text {. }
$$

Also, the velocity field must be invariant under all the symmetries, i.e.:

$$
k^{\mu} u^{\alpha},_{\mu}-u^{\mu} k^{\alpha}{ }_{\mu}=0 .
$$


But in our case $u^{\alpha}=\delta_{0}^{\alpha}$, which means that all the components of $k^{\alpha}$ must be independent of $t$. The remaining Killing equations reduce then to:

$$
\begin{array}{r}
k^{\mu} g_{11, \mu}+2 k^{1},_{1} g_{11}=0, \\
k^{2},_{1} g_{22}+k^{1},_{2} g_{11}=0, \\
k^{3},_{1} g_{33}+k^{1},_{3} g_{11}=0, \\
k^{\mu} g_{22, \mu}+2 k^{2},_{2} g_{22}=0, \\
k^{3},_{2}+k^{2},_{3}=0, \\
k^{\mu} g_{33, \mu}+2 k^{3},_{3} g_{33}=0,
\end{array}
$$

where $x^{1}=z, x^{2}=x, x^{3}=y$. We assume that:

$$
\left(g_{22} / g_{11}\right)_{t} \neq 0 \neq\left(g_{33} / g_{11}\right){ }_{t}
$$

because otherwise the shear is zero and the solution reduces to an $\mathrm{R}-\mathrm{W}$ metric. With (B.10) assumed, eqs. (B.5) and (B.6) imply:

$$
k^{2},_{1}=k^{3},_{1}=k^{1},_{2}=k^{1},_{3}=0 .
$$

Hence:

$$
k^{1}=A(z)
$$

Eq. (B.8) now implies that there exists a function $b(x, y)$ such that:

$$
k^{2}=b, x, \quad k^{3}=-b,,_{y}
$$

and since $g_{22}=g_{33}$, eqs. (B.7) and (B.9) imply:

$$
b, x x+b, y y=0 .
$$

Eq. (B.7) now implies:

$$
\left(-k x b,_{x}+k y b,_{y}\right) /\left[1+\frac{1}{4} k\left(x^{2}+y^{2}\right)\right]-2 b,_{y y}=0 .
$$

We have thus reduced the Killing equations to (B.4) and (B.12) - (B.15). The equations (B.14) - (B.15) define the following solution:

$$
b=\frac{k}{6}\left[c_{3}\left(x^{3}-3 x y^{2}\right)+c_{1}\left(3 x^{2} y-y^{3}\right)\right]+c_{2} x y+2 c_{3} x-2 c_{1} y+c_{4},
$$


where $c_{1}, \ldots, c_{4}$ are constants.

Now suppose that $A \neq 0$. Since the coordinate $z$ can be arbitrarily transformed, we can choose it so that $A=1$.

In the following, the cases $k \neq 0$ and $k=0$ have to be considered separately. When $k \neq 0$, eq. (B.4) implies:

$$
\lambda / \Phi=g(z)+f(t)
$$

where $g$ and $f$ are functions of one variable each (to be determined further). Since we already assumed that $\left(g_{22} / g_{11}\right)_{t} \neq 0$, we have $f_{, t} \neq 0$ automatically. It follows then from (4.7) that:

$$
\begin{gathered}
g=(C-U) / k, C=\text { const } \\
\Phi^{2} f_{t t}+3 \Phi \Phi,_{t} f_{, t}-k f=C
\end{gathered}
$$

and:

$$
\begin{gathered}
\left(U,_{z} / k-2 c_{3} V_{1}-2 c_{1} V_{2}\right) \frac{1}{4} k\left(x^{2}+y^{2}\right)+ \\
+\left(V_{1, z}+2 c_{3} U-c_{2} V_{2}\right) x+\left(V_{2, z}+c_{2} V_{1}+2 c_{1} U\right) y+ \\
-U,_{z} / k+2 c_{3} V_{1}+2 c_{1} V_{2}=0 .
\end{gathered}
$$

The coefficients of $\left(x^{2}+y^{2}\right), x, y$ and the term independent of $x$ and $y$ must vanish separately. This implies:

$$
c_{2} U, z / k-2 c_{1} V_{1, z}+2 c_{3} V_{2, z}=0
$$

Unless this specific relation among the functions $\left\{U, V_{1}, V_{2}\right\}$ exists, there can be no symmetries. Since in general $U, V_{1}$ and $V_{2}$ are just arbitrary, eq. (B.21) implies $c_{1}=c_{2}=c_{3}=0$. Then (B.20) will be violated unless $U, V_{1}$ and $V_{2}$ are constants. Hence, the assumption $A \neq 0$ leads to a contradiction, and so in general $A=0$ when $k \neq 0$.

When $A=1$ and $k=0$, eq. (B.4) implies at once that $U=$ const. In addition, (B.17) reappears, and an analog of (B.20) follows in which $(U, z / k)$ is replaced by $g, z$. Consequently, two differential equations are imposed on $V_{1}$ and $V_{2}$. The equations imply specific forms of these two functions. Hence, with arbitrary $U, V_{1}$ and $V_{2}$, again $A=0$ follows.

With $A=0$, another equation follows from (B.4) instead of (B.20), and it implies:

$$
\begin{gathered}
c_{3} V_{1}+c_{1} V_{2}=0, \\
2 c_{3} U-c_{2} V_{2}=0,
\end{gathered}
$$




$$
c_{2} V_{1}+2 c_{1} U=0
$$

which again do not hold for general $U, V_{1}$ and $V_{2}$ unless $c_{1}=c_{2}=c_{3}=0$. But then, $k^{\alpha}=0$ and there are no symmetries.

Note that there can be no symmetries even with the very special form of $\lambda$ given by (B.17) - (B.18), nor even with $U=0$ as long as $V_{1}$ and $V_{2}$ are arbitrary and the shear is nonzero. Hence, the two functions of $z$ that are in general hidden in $\lambda$ (see eq. (4.36)) do not matter in determining symmetries (this is the point that was not clear in the paper by Bonnor et al. [18]).

\section{Appendix C}

The case investigated by Quevedo and Sussman [7].

Quevedo and Sussman noticed that (4.11) is fulfilled when:

$$
F=X, \quad \lambda=\Phi B_{0} U(z),
$$

where $B_{0}(t)$ is a function of $t$ which, in virtue of (4.7), must obey:

$$
B_{0, t t}+3(\Phi, t / \Phi) B_{0, t}-\left(k / \Phi^{2}\right) B_{0}-1 / \Phi^{2}=0
$$

(differences between (C.2) and the corresponding equation in Ref. [7] result from our choice $W=0)$. Then:

$$
E=(U / \Phi)\left(\Phi \Phi,{ }_{t} B_{0, t}-k B_{0}-1\right)
$$

and so (C.1) is the subcase of (4.23) given by:

$$
A=1, \quad \psi=-\Phi B_{0} /\left(\Phi \Phi,{ }_{t} B_{0, t}-k B_{0}-1\right) .
$$

Substituting this $\psi$ into (A.1) and (A.2) one can verify that $F_{2}=0$ follows. The following formulae are helpful in the verification:

$$
\begin{aligned}
& K(t)=\Phi_{,} / \Phi+B_{0, t} / B_{0}-\left(B_{0} \Phi \Phi, t\right)^{-1}, \\
& e^{-\int K d t}=\left(B_{0} \Phi\right)^{-1} e^{\int\left(B_{0} \Phi \Phi,{ }_{t}\right)^{-1} d t} \\
& \int(1 / \Phi, t) e^{-\int K d t} d t=\int\left(B_{0} \Phi \Phi,{ }_{t}\right)^{-1} e^{\int\left(B_{0} \Phi \Phi, t\right)^{-1}} d t d t=e^{\int\left(B_{0} \Phi \Phi,{ }^{-1}\right)^{-1} d t} .
\end{aligned}
$$


Using (C.5) - (C.7) one can also verify that with $J=H=0$ the function $\lambda$ given by (4.28) has indeed the form (C.1) in this case.

\section{Appendix D}

\section{The degenerate solution of (5.17).}

If the coefficient of $(F, X / F)$ in (5.17) is zero, then necessarily

$$
\left(E,_{Z} / E\right)_{,}+3(\Phi, Z / \Phi)_{,}=0
$$

what integrates to:

$$
E=f_{1}(Z) f_{2}(t) / \Phi^{3},
$$

where $f_{1}$ and $f_{2}$ are arbitrary functions. Using (D.1) we see that the coefficient of $F,{ }_{X}$ is zero when:

$$
(\Phi, Z / \Phi){ }_{,} f_{1, Z} / f-6(\Phi, Z / \Phi)(\Phi, Z / \Phi)_{,}-\left(\Phi,,_{Z} / \Phi\right),{ }_{Z t}=0 .
$$

A first integral of this is:

$$
\left(\Phi,,_{Z} / \Phi\right)_{,}=f_{1}(Z) f_{3}(t) / \Phi^{6}
$$

Note that we can assume $f_{1} f_{3} \neq 0$ because otherwise the shear is zero (see Property III in sec. 5) and the R-W metrics result. If we now substitute eq. (5.8), the definition of $E$, into (D.1), then (D.1) and (D.3) will form a set of two partial differential equations to be obeyed by $\Phi$. The further procedure is this: substitute $\Phi_{, t Z}$ from (D.3) into (D.1), the result is:

$$
\Phi,_{t}=k \Phi^{4} \Phi,_{Z} /\left(f_{1} f_{3}\right)-k,_{Z} \Phi^{5} /\left(2 f_{1} f_{3}\right)+f_{2} \Phi /\left(2 f_{3}\right) .
$$

Now differentiate (D.4) by $Z$ and substitute for $\Phi_{, t Z}$ from (D.3) and for $\Phi_{, t}$ from (D.4). The result is:

$k \Phi,,_{Z Z}=-3 k \Phi,_{Z}^{2} / \Phi+k f_{1, Z} \Phi,,_{Z} / f_{1}+\frac{1}{2} k,_{Z Z} / \Phi+k,_{Z} \Phi,_{Z}-\frac{1}{2} k,_{Z} f_{1, Z} \Phi / f+f_{1}^{2} f_{3}^{2} / \Phi^{9}$.

It follows that if $k=0$, then $f_{1} f_{3}=0$, i.e. an $\mathrm{R}$-W metric results (see above). If $k \neq 0$, then we find $\Phi,{ }_{t Z Z}$ from (D.5), equate the result to $\Phi,{ }_{t Z Z}$ found from (D.3), use (D.3) to eliminate $\Phi_{, t Z},\left(\right.$ D.4) to eliminate $\Phi_{, t}$, and (D.5) to eliminate $\Phi, Z Z$. The result is:

$$
\Phi, Z=\frac{1}{2} k,_{Z} \Phi / k+\frac{1}{6} f_{1} f_{3, t} /\left(k \Phi^{3}\right)-\frac{5}{12} f_{1} f_{2} /\left(k \Phi^{3}\right) .
$$


Using this in (D.4) we get:

$$
\Phi, t=\frac{1}{6}\left(f_{3, t} / f_{3}\right) \Phi+\frac{1}{12}\left(f_{2} / f_{3}\right) \Phi
$$

This implies $(\ln \Phi), t Z=0$, i.e. an R-W model (Property III in sec. 5).

Note that $(E, Z / E),_{t}+3\left(\Phi,,_{Z} / \Phi\right),_{t}=0$ and $F,_{X}=0$ cannot hold simultaneously. To see this, substitute both these equations into (5.16) and differentiate the result by $X$ - a clear contradiction follows.

\section{Appendix E}

\section{Consistency of (5.21) with (5.5).}

From (5.21) we have:

$$
\Phi{ }_{t}^{2}+k=-g_{1} /(3 A \Phi)-B \Phi^{2} /(3 A)
$$

We differentiate this by $t$, use (5.5) to eliminate $\Phi, t$ from the result and (E.1) to eliminate $\Phi,{ }_{t}^{2}$. The resulting equation is:

$$
g_{1} A,{ }_{t} /\left(3 A^{2} \Phi^{2}\right)-\frac{1}{3}(B / A),_{t} \Phi=(B / A-\kappa p) \Phi,{ }_{t} .
$$

Suppose that $B / A=\kappa p$. Then there are two possibilities:

I. $g_{1} A,_{t}=0=(B / A){ }_{t}$. In this case $\kappa p=B / A=$ const and the thermodynamics becomes trivial.

II. $(B / A),_{t} \neq 0$. Then (E.2) can be solved for $\Phi$, and the solution has the property $(\ln \Phi){ }_{t} Z=0$. This is an R-W model (see Property III in sec. 5).

Hence, we can assume $B / A-\kappa p \neq 0$. Then, from (E.2):

$$
\Phi,{ }_{t}=g_{1} A,{ }_{t} /\left[3 A^{2} \Phi^{2}(B / A-\kappa p)\right]-\frac{1}{3}(B / A),{ }_{t} \Phi /(B / A-\kappa p) .
$$

This we differentiate by $t$, use (5.5) to eliminate $\Phi, t t,\left(\right.$ E.1) to eliminate $\Phi_{,}{ }_{t}^{2}$ and (E.3) to eliminate $\Phi, t$. The result is:

$$
\begin{gathered}
-\frac{2}{9} h_{1}^{2}\left(g_{1} / \Phi^{3}\right)^{2}+\left[-\frac{1}{9} h_{1} h_{2}-1 /(6 A)+\frac{1}{3} h_{1, t}\right] g_{1} / \Phi^{3} \\
+\frac{1}{3} h_{2, t}+\frac{1}{9} h_{2}^{2}-\frac{1}{6}(B / A)+\frac{1}{2} \kappa p=0,
\end{gathered}
$$


where:

$$
h_{1}(t):=A,_{t} /\left[A^{2}(B / A-\kappa p)\right], \quad h_{2}(t):=-(B / A){ }_{t} /(B / A-\kappa p) .
$$

Note that the coefficients of $\left(g_{1} / \Phi^{3}\right)^{2}$ and $\left(g_{1} / \Phi^{3}\right)$ in (E.4) cannot vanish simultaneously because this would lead to a contradiction. Therefore, there are two possibilities to consider:

I. $g_{1}=0$ so that $\left(\right.$ E.4) does not determine $\Phi$. In this case $\left(\right.$ E.3) implies $(\ln \Phi),{ }_{t} Z=0$ what is an R-W limit (see Property III in sec. 5).

II. $g_{1} \neq 0$. Then (E.4) can be solved for $\left(g_{1} / \Phi^{3}\right)$. However, the solution has the form $g_{1} / \Phi^{3}=[a$ function of $t$ only $]$, hence again $(\ln \Phi){ }_{t Z}=0$ and an R-W model results. This proves the concluding statement of sec. 5 .

\section{REFERENCES}

[1] C. Bona, B. Coll, Gen. Rel. Grav. 20, 297 (1988).

[2] H. Stephani, Commun. Math. Phys. 4, 137 (1967).

[3] A. Krasiński, Gen. Rel. Grav. 13, 1021 (1981).

[4] A. Krasiński, Gen. Rel. Grav. 15, 673 (1983).

[5] A. Krasiński, in: The Big Bang and Georges Lemaitre. Edited by A. Berger. D. Reidel Publishing Company, Dordrecht 1984, p. 63.

[6] B. Coll, J. J. Ferrando, J. Math. Phys. 30, 2918 (1989).

[7] H. Quevedo, R. Sussman, Class. Quantum Grav. 12, 859 (1995).

[8] D. Szafron, J. Math. Phys. 18, 1673 (1977).

[9] H. Quevedo and R. A. Sussman, J. Math. Phys. 36, 1365 (1995).

[10] A. Krasiński, Inhomogeneous cosmological models. Cambridge University Press, in press (at present available as a preprint under the title Physics in an inhomogeneous Universe).

[11] H. Stephani, Class. Quantum Grav. 4, 125 (1987).

[12] P. Szekeres, Commun. Math. Phys. 41, 55 (1975); Phys. Rev. D12, 2941 (1975).

[13] D. Kramer, H. Stephani, E. Herlt, M. A. H. MacCallum, Exact solutions of the Einstein field equations. Cambridge University Press 1980.

[14] C. Bona and B. Coll, C. R. Acad. Sci. Paris, 1301, 613 (1985).

[15] R. Kantowski, R. K. Sachs, J. Math. Phys. 7, 443 (1966). 
[16] A. S. Kompaneets, A. S. Chernov, ZhETF 47, 1939 (1964) [Sov. Phys. JETP 20, $1303(1965)]$.

[17] A. Spero, D. A. Szafron, J. Math. Phys. 19, 1536 (1978).

[18] W. B. Bonnor, A. H. Sulaiman, N. Tomimura, Gen. Rel. Grav. 8, 549 (1977).

[19] S. W. Goode, J. Wainwright, Phys. Rev. D26, 3315 (1982).

[20] Maple's Reference Manual (University of Waterloo, Canada, 1994)

[21] A. Krasiński, Gen. Rel. Grav. 25, 165 (1993). 